\title{
Tumour endothelial marker-8 in wound healing and its impact on the proliferation and migration of keratinocytes
}

\author{
SHEILA C. WANG ${ }^{1,2}$, LIN YE ${ }^{1}$, ANDREW J. SANDERS ${ }^{1}$, FIONA RUGE ${ }^{1,2}$, \\ KEITH G. HARDING ${ }^{2}$ and WEN G. JIANG ${ }^{1}$ \\ ${ }^{1}$ Cardiff China Medical Research Collaborative (CCMRC) and ${ }^{2}$ Department of Wound Healing, \\ Cardiff University School of Medicine, Cardiff CF14 4XN, UK
}

Received August 26, 2015; Accepted October 13, 2015

DOI: $10.3892 /$ ijmm.2015.2434

\begin{abstract}
Chronic wound management represents a significant burden on healthcare systems and negatively impacts on the quality of patient life. New strategies to understand and identify wounds that will not heal in a normal manner are required. Tumour endothelial marker-8 (TEM-8) has been implicated in the wound healing and angiogenesis processes. TEM- 8 expression was examined at the transcript level in a cohort of acute $(n=10)$ and chronic $(n=14)$ wounds and in normal skin $(n=10)$. Protein analysis of TEM- 8 was also undertaken for this cohort using immunohistochemistry (IHC). TEM-8 impact on keratinocyte cell growth and migration was assessed following TEM-8 ribozyme transgene transfection of human $\mathrm{HaCaT}$ keratinocytes using cell growth and electric cell-substrate impedance sensing (ECIS)-based assays. Expression of TEM-8 was observed to be increased in acute wounds compared to chronic wounds and normal skin using quantitative polymerase chain reaction transcript analysis and IHC staining of wound tissues. Knockdown of TEM- 8 in HaCaT cells, using two independent ribozyme transgenes, resulted in significant decreases in cell growth as well as reductions in the rate of migration assessed using an ECIS-based system. TEM-8 may be differentially expressed between wound types and loss of this molecule impacts $\mathrm{HaCaT}$ growth and migration, potentially implicating this molecule as a factor involved in successful progression of wound healing.
\end{abstract}

\section{Introduction}

The management of chronic wounds is a significant drain on healthcare resources; the cost of wounds to the United

Correspondence to: Professor Wen G. Jiang, Cardiff China Medical Research Collaborative (CCMRC), Cardiff University School of Medicine, Henry Wellcome Building, Heath Park, Cardiff CF14 4XN, UK

E-mail: jiangw@cf.ac.uk

Key words: tumour endothelial marker-8, wound healing, migration, chronic wound, acute wound
Kingdom healthcare system alone is estimated to be $\sim £ 1$ billion per year (1), notwithstanding the economic loss and impaired quality of life experienced by people with chronic ulcers. Identification of the molecular factors underlying chronic and acute wounds is critical for developing improved tailored treatments for wound healing.

In acute wounds caused by trauma to intact skin, normal wound healing involves three overlapping dynamic phases of inflammation (lasting 1-3 days), proliferation (lasting 3-14 days) and remodelling (can last up to several months) $(2,3)$. However, in chronic wounds, $70 \%$ of which is represented by venous leg ulcers, often underlying disease states reduce healing and this dynamic process does not proceed in an orderly or timely manner to produce anatomic and functional skin integrity within 3 months, as observed in acute wounds (4).

The biology of skin healing in acute and chronic wounds involves complex interactions between epidermal and dermal cells, the extracellular matrix and plasma-derived proteins (5). Currently, research is focusing on understanding the role of angiogenesis in physiological and pathological processes, such as inflammation, wound healing and tumour angiogenesis.

Tumour endothelial marker-8 (TEM-8) is a highly conserved type 1 transmembrane protein that was originally identified based on its overexpression in the endothelial cells lining the tumour vasculature of human colorectal cancer (6). The present understanding of the physiological function of TEM- 8 is limited; the high level of conservation of TEM- 8 among different species suggests that TEM-8 has a fundamental role in normal physiology, as well as pathological processes. TEM- 8 has been found to bind to collagens and promote migration of endothelial cells in vitro $(7,8)$, and thus have a potential role in angiogenesis (9-11) and wound healing.

TEM-8 is upregulated in tumour vasculature in mice and humans $(7,12,13)$, and is also expressed by tumour cells themselves in certain cancer types $(12,14,15)$. However, TEM- 8 could not be detected in the angiogenic corpus luteum of human ovaries $(6,7)$ and thus presents itself as a unique target for selectively blocking pathological angiogenesis. Previous studies have shown that the genetic disruption and antibody-mediated disruption of TEM-8 in mice inhibited tumour angiogenesis and growth, but did not perturb acute wound healing observed for $\leq 7$ days $(16,17)$. However, the role of TEM- 8 in chronic non-healing wounds has not been 
investigated previously. Inhibition of angiogenesis is known to impair wound healing (18-20) and previous studies have revealed potential microenvironmental factors, specifically a reduction in tissue growth factors, which is known to impair healing (21), have now been shown to also induce TEM- 8 expression $(16,22)$.

The present study investigated the role of TEM-8 in wound healing processes, specifically its expression in clinical chronic wound samples and its influence on $\mathrm{HaCaT}$ growth and migrational rates.

\section{Materials and methods}

Materials. A universal immunohistochemical kit, Elite ABC kit, was purchased from Vector Laboratories (Peterborough, UK). The total RNA isolation reagent was purchased from Sigma-Aldrich (Dorset, UK) and reverse transcription kits (iScript) were obtained from Bio-Rad Laboratories (Hemel Hempstead, UK).

Skin biopsies. Skin biopsies were obtained from patients attending the University Hospital of Wales (UHW, Cardiff, UK) wound healing clinic, as described previously $(23,24)$. Ethical approval was granted by the Local Research Ethics Committee. Written informed consent was obtained from each patient.

Chronic wound tissue. Biopsies from 14 patients with chronic leg ulcers were used during the study. Venous disease was diagnosed by duplex ultrasonography and all the wounds were present for $\geq 6$ months, with no evidence of healing occurring 6 weeks before biopsy. The wounds had a minimum area of $4 \mathrm{~cm}^{2}$ prior to biopsy and had no clinical indications of infection. Using an aseptic technique, 6-mm punch biopsies were removed, following the application of local anesthetic (1\% lidocaine), from the wound margin, incorporating epidermis and dermis at the wound edge with adjacent granulation tissue.

Acute wound tissue. Single wedge biopsies were obtained from 10 patients with acute surgical wounds subsequent to undergoing excision of pilonoidal disease. These wounds were judged to be clinically noninfected. The biopsies were obtained from the edge of the healing wound within 6 weeks from the surgical excision.

Normal skin tissue. To provide a comparison to wound tissue, normal skin tissue was also examined. Under local anesthetic, 3-mm punch biopsies were removed from the inner aspect of the upper arm of 10 healthy volunteers working within the Wound Healing Research Unit (School of Medicine, Cardiff University, Cardiff, Wales).

Immunohistochemical staining. Frozen sections from wound tissues were first fixed in an acetone/methanol solution and rehydrated in wash buffer (MenaPath Autowash buffer; A. Menarini Diagnostics, Berkshire, UK) prior to placing the samples into a wash buffer solution containing $10 \%$ horse serum to aid in the blocking of non-specific antigen binding. An avidin/biotin complex (ABC) immunohistochemistry (IHC) kit (Vector Laboratories, Nottingham, UK) was used in accordance with the manufacturer's protocol. A polyclonal antibody to TEM-8, previously generated (9), was used and diluted in a buffer that contained $1 \%$ horse serum and $0.1 \%$ Tween-20 at 1:40 dilution. After a 1-h incubation period with the primary antibody, the slides were washed 4 times in a washing buffer and a universal biotinylated secondary antibody was added for $30 \mathrm{~min}$. Following washing, avidin and biotin were added through the addition of the ABC complex. A DAB colour developing system was used to indirectly detect protein staining. Sections were dehydrated through a series of graded alcohols, cleared in xylene, mounted and evaluated on an Olympus microscope equipped with a digital camera (Olympus, Southend-on-Sea, UK).

HaCaT cell line and culture conditions. The HaCaT human keratinocyte cell line was purchased from the German Cancer Research Institute (Heidelberg, Germany). Cells were maintained in Dulbecco's modified Eagle's medium supplemented with penicillin, streptomycin and $10 \%$ fetal calf serum (PAA Laboratories Ltd., Somerset, UK). The cells were incubated at $37^{\circ} \mathrm{C}, 5 \% \mathrm{CO}_{2}$ and $95 \%$ humidity.

RNA extraction and reverse transcription-polymerase chain reaction $(R T-P C R)$. Cells were grown to confluence in a $25-\mathrm{cm}^{2}$ flask prior to RNA extraction using the total RNA isolation reagent in accordance with the manufacturer's protocol. Sample RNA was quantified using a spectrophotometer (WPA UV 1101; Biotech Photometer, Cambridge, UK) and standardized to a concentration of $500 \mathrm{ng}$ prior to being used as a template to reverse transcribe cDNA using an iScript cDNA synthesis kit (Bio-Rad Laboratories). Following cDNA synthesis, samples were probed using glyceraldehyde 3-phosphate dehydrogenase (GAPDH) primers to check the cDNA quality and confirm uniform sample cDNA levels, together with those specific for TEM-8 transcript (Table I) as previously reported $(9,11)$.

Conventional RT-PCR primers were designed using Beacon designer Software (Beacon Designer, Palo Alto, CA, USA), to allow amplification of regions that have no overlap with known genes and span at least one intron. Primers were synthesized by Invitrogen (Paisley, UK). Conditions for conventional RT-PCR to amplify transcripts of TEM- 8 were: $94^{\circ} \mathrm{C}$ for $40 \mathrm{sec}, 54^{\circ} \mathrm{C}$ for $30 \mathrm{sec}, 72^{\circ} \mathrm{C}$ for $50 \mathrm{sec}$ and a final extension phase of $10 \mathrm{~min}$ for 34-36 cycles, with GAPDH used as the reference gene. The PCR products were separated on a $0.8 \%$ agarose gel and stained with ethidium bromide prior to examination under UV light.

RT-quantitative PCR (RT-qPCR). RT-qPCR was used to determine the transcript expression levels of TEM- 8 in wound tissues. This methodology has been reported previously (25). Briefly, the iCycler IQ system (Bio-Rad, Camberley, UK) was used to quantify the transcript level of TEM- 8 within each of the clinical samples. Results are provided as number of transcripts per microlitre and are based on an internal standard run and amplified in conjunction with the samples. Normalisation of samples was achieved through comparison of sample GAPDH levels. The Amplifluor system (Intergen Inc., New York, NY, USA) was used in conjunction with a universal probe (UniPrimer), which recognised a specific sequence ( $\mathrm{z}$ sequence), incorporated into the primers. RT-qPCR conditions were as follows: $95^{\circ} \mathrm{C}$ for $15 \mathrm{sec}, 54^{\circ} \mathrm{C}$ for $20 \mathrm{sec}$ and $60^{\circ} \mathrm{C}$ for $60 \mathrm{sec}$. 
Table I. Primer and ribozyme sequences.

\begin{tabular}{lll}
\hline Primer/sequence & \multicolumn{1}{c}{ Forward } & \multicolumn{1}{c}{ Reverse } \\
\hline TEM-8 ribozyme 1 & ctgcagggggccatagagacggctgatgagccgtga & actagtccacagctattatgtgtttcgtcctcacggac \\
TEM-8 ribozyme 3 & gtgcagacttcttcaaaattgagtggatctgatgactccetga & actagtttcaggctctgcaaggcatttcgtcctcacgga \\
TEM-8 conventional & cattcaagttgtcgtgaga & gacgcatattgttgttgaga \\
TEM-8 quantitative & acagggtcctctgcagctt & actgaacctgaccgtacactttcatgccaacttgttt \\
GAPDH conventional & agcttgtcatcaatggaaat & cttcaccaccttcttgatgt \\
GAPDH quantitative & ctgagtacgtcgtggagtc & actgaacctgaccgtacacagagatgatgaccettttg
\end{tabular}

actgaacctgaccgtacaca represents the z sequence. TEM-8, tumour endothelial marker-8; GAPDH, glyceraldehyde 3-phosphate dehydrogenase.

TEM-8 knockdown in the human HaCaT keratinocyte cell line. Hammerhead ribozyme transgenes, specifically targeted to TEM-8 transcripts were constructed based on the secondary structure of TEM- 8 mRNA, as described previously (10) (Table I). Following the design and synthesis by Invitrogen, two separate ribozymes, targeting different regions of the TEM- 8 transcript, were cloned into a mammalian pEF6/His TOPO vector (Invitrogen) and transfected into $\mathrm{HaCaT}$ cells, as previously reported (10). Following a period of blasticidin selection $(5 \mu \mathrm{g} / \mathrm{ml})$, the cells were maintained in media containing $0.5 \mu \mathrm{g} / \mathrm{ml}$ blasticidin. This process allowed the generation of stably transfected HaCaT cells containing the ribozyme transgene and expressing reduced TEM- 8 levels $\left(\mathrm{HaCaT}^{\triangle \mathrm{TEM}-8 \text { ribl }}\right.$ and $\mathrm{HaCaT}^{\Delta \mathrm{TEM}-8 \mathrm{rib3}}$ ) and control $\mathrm{HaCaT}$ cells transfected with a closed pEF6 plasmid $\left(\mathrm{HaCaT}^{\mathrm{pEF} 6}\right)$. Suppression of TEM-8 expression in $\mathrm{HaCaT}^{\Delta \mathrm{TEM}-8 \mathrm{rib} 1}$ and $\mathrm{HaCaT}^{\Delta \mathrm{TEM}-8 \text { rib3 }}$ cells was verified, in comparison to $\mathrm{HaCaT}^{\mathrm{EF} 6}$ controls, using RT-PCR.

In vitro growth assay. The effect of TEM-8 suppression on $\mathrm{HaCaT}$ cell growth rates was assessed using an in vitro growth assay. Cells were seeded at a density of 3,000 cells/well in 96-well plates. Triplicate plates were set up and incubated for 3- and 5-day periods before analyses. Following incubation, the plates were fixed in $4 \%$ formaldehyde $(\mathrm{v} / \mathrm{v})$ and stained with $0.5 \%(\mathrm{w} / \mathrm{v})$ crystal violet and were subsequently treated with $10 \%$ acetic acid (v/v), prior to colorimetric detection of cell density by spectrophotometric analysis at $540 \mathrm{~nm}$ using a Bio-Tek ELx800 mutliplate reader (Bio-Tek Instruments Inc., Winnoski, VT, USA).

Electric cell-substrate impedance sensing (ECIS) analysis of HaCaT migration. The ECIS 9600 system (Applied Biophysics Inc., Troy, NJ, USA) was used to detect and track HaCaT cell migration, as described previously $(26,27)$. Briefly, cells were simultaneously plated in ECIS 8W10 arrays and incubated until a confluent monolayer had formed over the array electrodes. This monolayer was subsequently wounded electrically by applying $6 \mathrm{~V}$ for a 30 -sec time-period to create a simultaneous physical break in the cell monolayer of equal dimensions. The rate of change in resistance as cells migrated back onto the electrode, was subsequently monitored and measured using the ECIS software provided. Prior to use, ECIS arrays were treated with L-cysteine solution for $40 \mathrm{~min}$ followed by several washes with complete medium to remove any particles present on the electrode.
Statistical analysis. The SigmaPlot 11 statistical package (Systat Software, San Jose, CA, USA) were used to identify statistical differences between the test groups using one-way analysis of variance (ANOVA) or ANOVA on RANKS tests. $\mathrm{P}<0.05$ was considered to indicate a statistically significant difference. All the in vitro functional assays were repeated a minimum of three times.

\section{Results}

TEM-8 expression is decreased in non-healing chronic venous leg ulcers compared to acute wounds. TEM-8 is minimally expressed in all layers of the epidermis of normal skin. However, in acute wounds, expression of TEM- 8 increases within the cytoplasm of keratinocytes and endothelial cells, consistent with its putative role in angiogenesis. Expression was also increased in chronic wounds compared to normal skin, but is lower compared to the acute wounds (Fig. 1A-C).

Consistent with the immunohistochemical analyses, RT-qPCR detection and normalisation of TEM-8 levels also revealed higher TEM-8 expression in acute (median, 3.964; IQR, 12.049-0.0445) and chronic (median, 1.755; IQR, 7.426-0.144) wounds and again, extremely low levels were observed in normal skin (median, 0.381; IQR, 1.996-0.0629), although no significant differences were observed within the group ( $\mathrm{P}>0.05)$ (Fig. 1D).

Suppression of TEM-8 expression reduces the growth rate of $\mathrm{HaCaT}$ keratinocytes. To determine the effect of reduced TEM-8 expression on keratinocyte cell growth, HaCaT keratinocytes were transfected with ribozyme transgenes specifically targeted to the TEM- 8 transcript to reduce TEM- 8 transcript levels within HaCaT cells, as assessed by RT-PCR (Fig. 2A).

In $\mathrm{HaCaT}$ keratinocytes in which TEM-8 expression was reduced, a decrease was also observed in the in vitro growth rate of the HaCaT cells (Fig. 2B). The growth rate of $\mathrm{HaCaT}^{\Delta \mathrm{TEM}-8 \text { rib1 }}$ and $\mathrm{HaCaT}^{\Delta \mathrm{TEM}-8 \text { rib3 }}$ cells over a 5-day incubation period was significantly lower compared to the $\mathrm{HaCaT}^{\mathrm{pEF} 6}$ control cells $(\mathrm{P}=0.004$ and $\mathrm{P}=0.014$, respectively). No significant differences between the groups were observed over a 3 -day incubation period $(\mathrm{P}>0.05)$.

Suppression of TEM-8 expression reduces the migrational rates of HaCaT cells. As TEM-8 expression was reduced in non-healing wounds compared to acute wounds and 
A

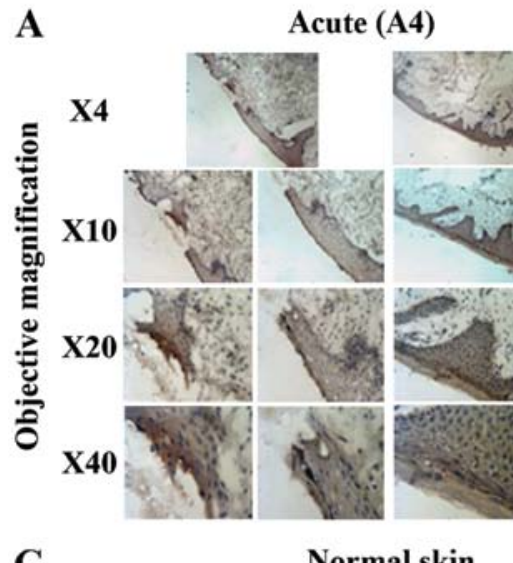

C

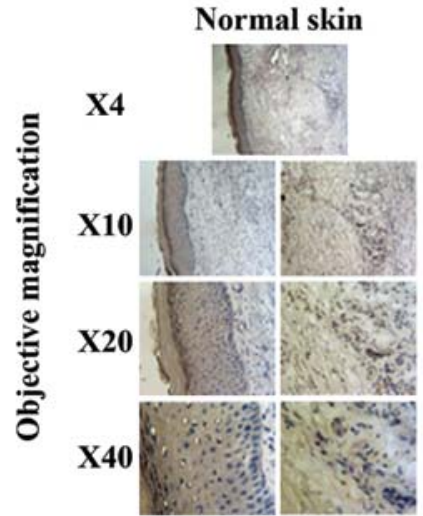

B

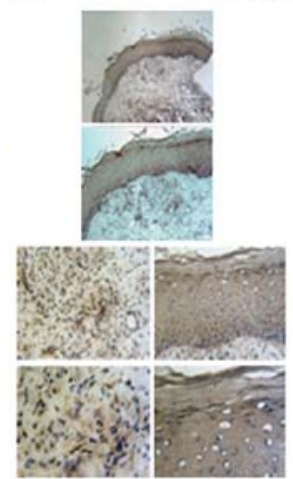

D

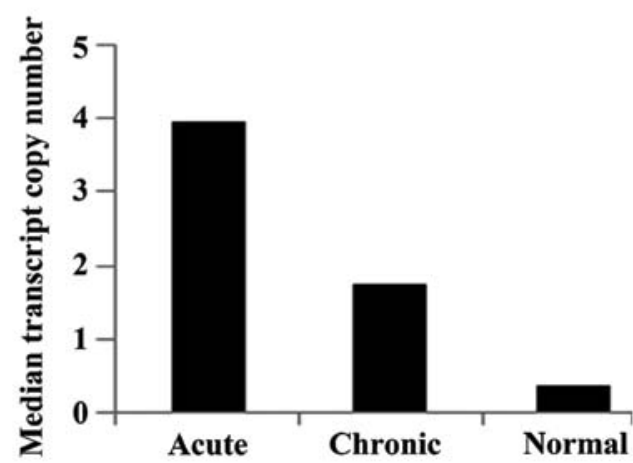

Figure 1. Expression of tumour endothelial marker-8 (TEM-8) in clinical wound and normal skin samples. Immunohistochemical staining analysis of TEM- 8 expression in (A) acute and (B) chronic wounds, and (C) normal skin tissue sections demonstrating increased cytoplasmic TEM-8 expression in keratinocytes and endothelial cells at the wound edge. (D) Quantitative transcript analysis of TEM-8 levels in acute and chronic wounds, and normal skin.

A
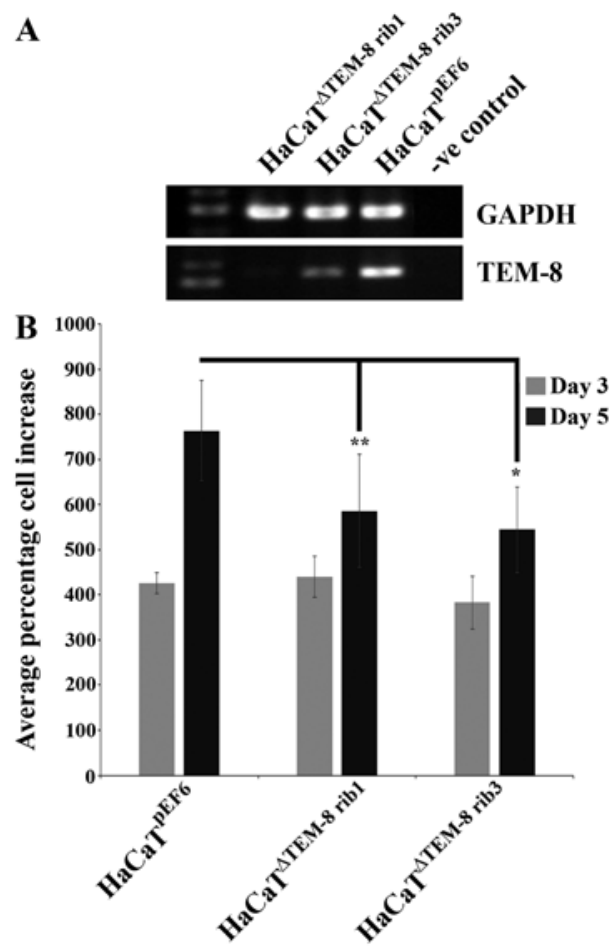

Figure 2. (A) Reverse transcription-polymerase chain reaction demonstrating successful knockdown of tumour endothelial marker-8 (TEM-8) following transfection of HaCaT cells with two independent TEM-8 ribozyme transgenes. (B) Knockdown of TEM-8, using either ribozyme transgene, significantly reduced cell growth rate over a 5-day incubation period in comparison to control cells. Representative data shown as average values \pm standard deviation, ${ }^{* *} \mathrm{P}<0.01$ and ${ }^{*} \mathrm{P}<0.05$. suppression of TEM-8 expression also reduced cell growth, the effects of TEM-8 knockdown on keratinocyte migration directly were investigated. An ECIS assay was carried out to examine the effects of TEM-8 suppression on $\mathrm{HaCaT}$ migration following electrical wounding (Fig. 3). Substantial differences between the migratory rates of $\mathrm{HaCaT}^{\mathrm{pEF} 6}$, $\mathrm{HaCaT}^{\Delta \mathrm{TEM}-8 \text { ribl }}$ and $\mathrm{HaCaT}^{\Delta \mathrm{TEM}-8 \mathrm{rib3}}$ were observed as the cell lines responded to re-colonise the wounded area of the array. $\mathrm{HaCaT}^{\mathrm{PF} 6}$ cells migrated at a steady rate following wounding as indicated by the increase in resistance recorded across the array. In contrast to this, $\mathrm{HaCaT}^{\mathrm{TTEM}-8 \text { ribl } 1}$ and $\mathrm{HaCaT}^{\mathrm{TTEM}-8 \text { rib3 }}$ cells showed a reduced capacity to migrate into the wound and recover the monolayer over the experimental time (Fig. 3A). Three-dimensional analysis further demonstrated this trend, showing changes in resistance across a range of tested frequencies and time for $\mathrm{HaCaT}^{\mathrm{EEF} 6}$ (Fig. 3B), $\mathrm{HaCaT}^{\triangle \mathrm{TEM}-8 \mathrm{ribl}}$ (Fig. 3C) and $\mathrm{HaCaT}^{\Delta \mathrm{TEM}-8 \text { rib3 }}$ (Fig. 3D).

\section{Discussion}

TEM- 8 is a cell surface receptor that has been shown to promote tumour angiogenesis. Given the importance of angiogenesis in tissue healing and repair, the role of TEM- 8 in acute and chronic wounds was investigated in the present study.

TEM-8 expression was significantly higher in acute wounds, measured at 6 weeks, and chronic wounds, measured at 6 months, compared to normal skin tissue. Targeting TEM-8 expression, using a ribozyme transgene system in the $\mathrm{HaCaT}$ keratinocyte cell line, resulted in decreased growth after 
A
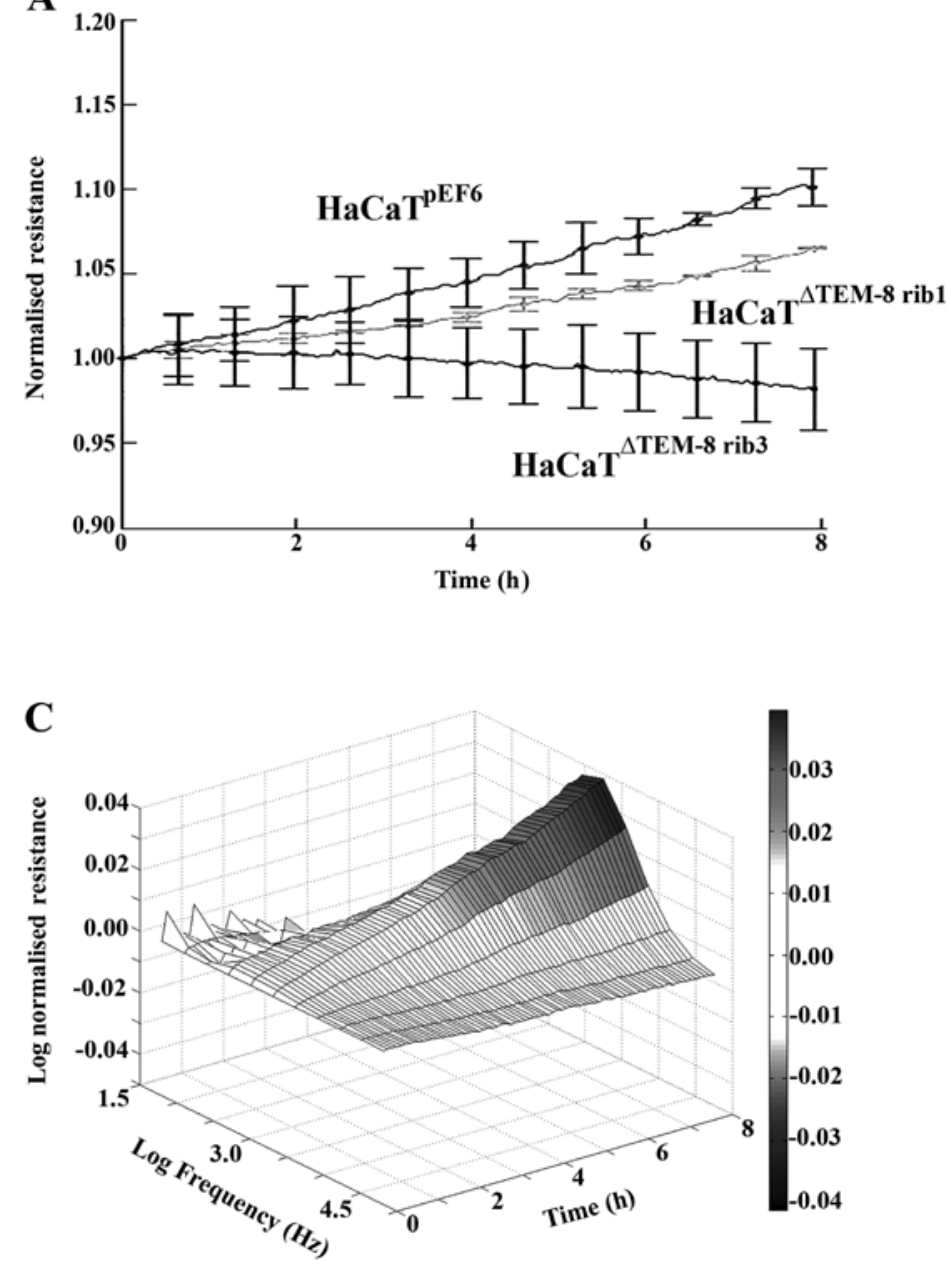

B

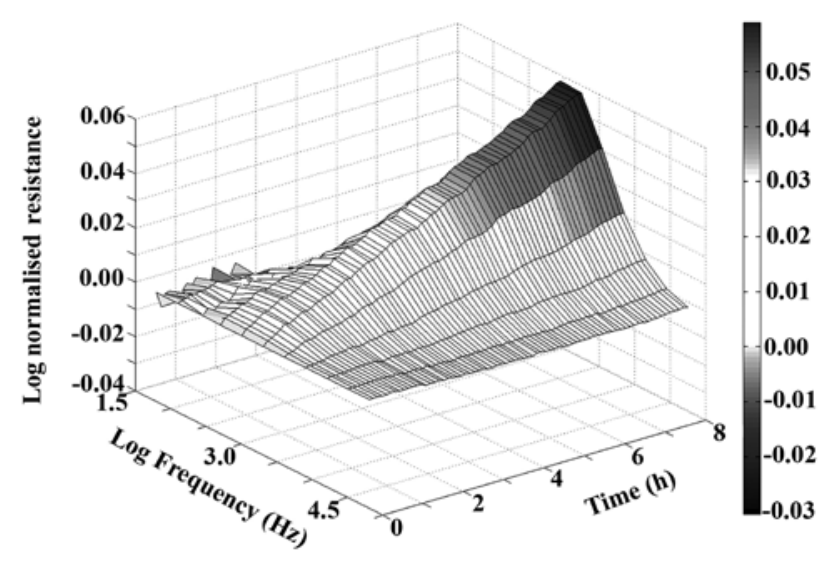

D

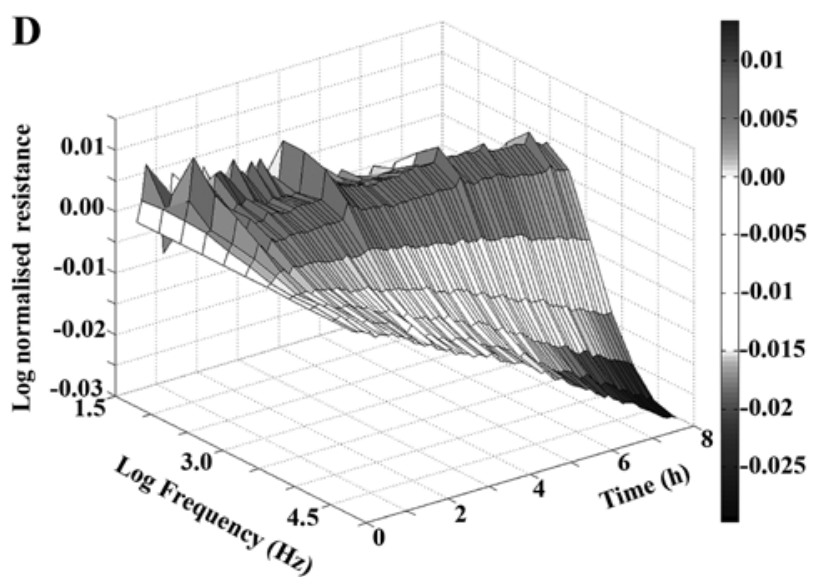

Figure 3. Electric cell-substrate impedance sensing analysis of cell migration. (A) Knockdown of tumour endothelial marker-8 (TEM-8) in HaCaT cells through transfection with either TEM- 8 ribozyme 1 or TEM- 8 ribozyme 3 reduced cell migration over an 8 -h period post electrical wounding in comparison to control cells. Three-dimensional modelling of changes in resistance following electrical wounding over a range of frequencies and time are shown for (B) $\mathrm{HaCaT}^{\mathrm{pEF}}$, (C) $\mathrm{HaCaT}^{\Delta \mathrm{TEM}-8 \text { rib1 }}$ and (D) $\mathrm{HaCaT}^{\Delta \mathrm{TEM}-8 \text { rib3 }}$. Representative data shown as average values \pm standard deviation.

5 days and decreased migration within hours in an ECIS-based assay. A similar role for TEM-8 in promoting cell migration was observed in previous studies demonstrating that TEM-8 mediates spreading of endothelial cells in vitro (8).

In other studies, TEM-8-KO and anti-TEM-8 antibodies did not appear to affect acute wound closure rates or the amount of vasculature present within the acute wound granulation tissue in mice measured at 5-7 days $(16,17)$. Therefore, it is important to note that wound healing was assessed at different times (days versus weeks/months) and the experiments reported in the present study were performed in vitro on single $\mathrm{HaCaT}$ cell lines, and therefore it is difficult to compare the direct effects on a single cell line with observed physiological responses in animal models, whereby multiple mechanisms and cells participate in wound healing.

Notably, TEM-8 expression was 2-fold higher in the acute versus chronic wounds, but nearly undetectable in normal skin tissue, consistent with studies by Chaudhary and St Croix, which demonstrated that local environmental stressors, including growth factor deprivation, reduced levels of which are commonly observed in non-healing wounds (21), led to TEM- 8 overexpression $(16,22)$.
It has been proposed that TEM- 8 may be involved in a transient stress-mediated response $(16,22)$, and numerous stressors, such as hypoxia and low levels of growth factors, exist in the chronic wound microenvironment $(18-21,28)$. Persistent elevated levels of TEM- 8 in chronic wounds suggest that TEM- 8 could have a role in pathological angiogenesis in non-healing wounds, as well as in tumours.

The notion that tumours represent 'unhealed wounds' is one of the oldest ideas in cancer biology (29) and it is perhaps not surprising that TEM- 8 could have a role in angiogenesis in both of these pathological processes.

An improved understanding of the mechanism and role TEM- 8 has in acute and chronic wounds requires further study and may allow for the development of more effective therapies for tumour angiogenesis and also chronic wound healing.

\section{Acknowledgements}

The authors would like to acknowledge the A4B Scheme of the Welsh Government, Ser Cymru, NRN life sciences research network and Cancer Research Wales for kindly supporting the present study. 


\section{References}

1. Harding KG: The future of wound healing. Wounds: Biology and Management 191-199, 1998.

2. Clark RAF: Biology of dermal wound repair. Dermatol Clin 11: 647-666, 1993

3. Singer AJ and Clark RAF: Cutaneous wound healing. N Engl J Med 341: 738-746, 1999.

4. Margolis DJ, Allen-Taylor L, Hoffstad O and Berlin JA: Diabetic neuropathic foot ulcers: Predicting which ones will not heal. Am J Med 115: 627-631, 2003.

5. Yamada KM and Clark RAF: Provisional matrix. The Molecular and Cellular Biology of Wound Repair 51-93, 1996.

6. St Croix B, Rago C, Velculescu V, Traverso G, Romans KE, Montgomery E, Lal A, Riggins GJ, Lengauer C, Vogelstein B et al: Genes expressed in human tumor endothelium. Science 289: 1197-1202, 2000.

7. Nanda A, Carson-Walter EB, Seaman S, Barber TD, Stampfl J, Singh S, Vogelstein B, Kinzler KW and St Croix B: TEM8 interacts with the cleaved C5 domain of collagen $\alpha 3$ (VI). Cancer Res 64: 817-820, 2004.

8. Werner E, Kowalczyk AP and Faundez V: Anthrax toxin receptor $1 /$ tumor endothelium marker 8 mediates cell spreading by coupling extracellular ligands to the actin cytoskeleton. J Biol Chem 281: 23227-23236, 2006.

9. Rmali KA, Al-Rawi MA, Parr C, Puntis MC and Jiang WG: Upregulation of tumour endothelial marker- 8 by interleukin-1beta and its impact in IL-1beta induced angiogenesis. Int J Mol Med 14: 75-80, 2004.

10. Rmali KA, Puntis MCA and Jiang WG: TEM-8 and tubule formation in endothelial cells, its potential role of its vW/TM domains. Biochem Biophys Res Commun 334: 231-238, 2005.

11. Rmali KA, Watkins G, Harrison G, Parr C, Puntis MCA and Jiang WG: Tumour endothelial marker 8 (TEM-8) in human colon cancer and its association with tumour progression. Eur J Surg Oncol 30: 948-953, 2004.

12. Carson-Walter EB, Watkins DN, Nanda A, Vogelstein B, Kinzler KW and St Croix B: Cell surface tumor endothelial markers are conserved in mice and humans. Cancer Res 61: 6649-6655, 2001.

13. Fernando $\mathrm{S}$ and Fletcher BS: Targeting tumor endothelial marker 8 in the tumor vasculature of colorectal carcinomas in mice. Cancer Res 69: 5126-5132, 2009.

14. Jinnin M, Medici D, Park L, Limaye N, Liu Y, Boscolo E, Bischoff J, Vikkula M, Boye E and Olsen BR: Suppressed NFAT-dependent VEGFR1 expression and constitutive VEGFR2 signaling in infantile hemangioma. Nat Med 14: 1236-1246, 2008.

15. Yang MY, Chaudhary A, Seaman S, Dunty J, Stevens J, Elzarrad MK, Frankel AE and St Croix B: The cell surface structure of tumor endothelial marker 8 (TEM8) is regulated by the actin cytoskeleton. Biochim Biophys Acta 1813: 39-49, 2011.
16. Chaudhary A, Hilton MB, Seaman S, Haines DC, Stevenson S, Lemotte PK, Tschantz WR, Zhang XM, Saha S, Fleming T, et al: TEM8/ANTXR1 blockade inhibits pathological angiogenesis and potentiates tumoricidal responses against multiple cancer types. Cancer Cell 21: 212-226, 2012.

17. Cullen M, Seaman S, Chaudhary A, Yang MY, Hilton MB, Logsdon D, Haines DC, Tessarollo L and St Croix B: Host-derived tumor endothelial marker 8 promotes the growth of melanoma. Cancer Res 69: 6021-6026, 2009.

18. Brem H, Ehrlich P, Tsakayannis D and Folkman J: Delay of wound healing by the angiogenesis inhibitor TNP-470. Surg Forum 48: 714-716, 1997.

19. Brem H, Tsakayannis D and Folkman J: Time dependent suppression of wound healing with the angiogenesis inhibitor, AGM-1470. J Cell Biol 115: 403a, 1991.

20. McGrath MH and Emery JM III: The effect of inhibition of angiogenesis in granulation tissue on wound healing and the fibroblast. Ann Plast Surg 15: 105-122, 1985.

21. Higley HR, Ksander GA, Gerhardt CO and Falanga V: Extravasation of macromolecules and possible trapping of transforming growth factor- $\beta$ in venous ulceration. $\mathrm{Br} \mathrm{J}$ Dermatol 132: 79-85, 1995.

22. Chaudhary A and St Croix B: Selective blockade of tumor angiogenesis. Cell Cycle 11: 2253-2259, 2012.

23. Conway K, Ruge F, Price P, Harding KG and Jiang WG: Hepatocyte growth factor regulation: An integral part of why wounds become chronic. Wound Repair Regen 15: 683-692, 2007.

24. Conway KP, Price P, Harding KG and Jiang WG: The role of vascular endothelial growth inhibitor in wound healing. Int Wound J 4: 55-64, 2007.

25. Jiang WG, Douglas-Jones A and Mansel RE: Expression of peroxisome-proliferator activated receptor-gamma (PPARgamma) and the PPARgamma co-activator, PGC-1, in human breast cancer correlates with clinical outcomes. Int $\mathrm{J}$ Cancer 106: 752-757, 2003.

26. Jiang WG, Martin TA, Lewis-Russell JM, Douglas-Jones A, Ye L and Mansel RE: Eplin-alpha expression in human breast cancer, the impact on cellular migration and clinical outcome. Mol Cancer 7: 71, 2008.

27. Keese CR, Wegener J, Walker SR and Giaever I: Electrical wound-healing assay for cells in vitro. Proc Natl Acad Sci USA 101: 1554-1559, 2004.

28. Hasan A, Murata H, Falabella A, Ochoa S, Zhou L, Badiavas E and Falanga V: Dermal fibroblasts from venous ulcers are unresponsive to the action of transforming growth factor- $\beta 1$. J Dermatol Sci 16: 59-66, 1997.

29. Dvorak HF: Tumors: Wounds that do not heal. Similarities between tumor stroma generation and wound healing. N Engl J Med 315: 1650-1659, 1986. 